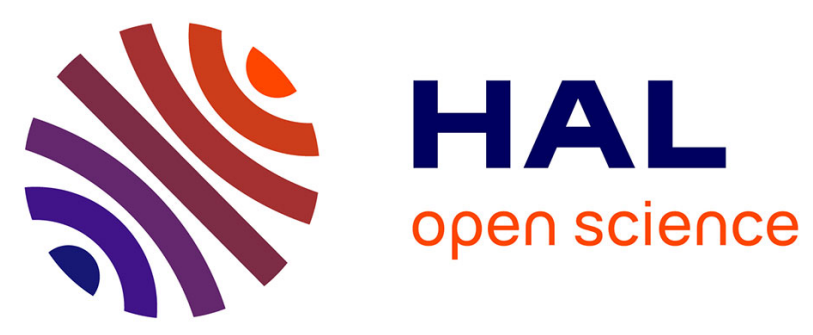

\title{
Penning trap mass spectrometry for nuclear structure studies
}

\author{
K. Blaum, D. Beck, M. Breitenfeldt, S. George, F. Herfurth, A. Herlert, A. \\ Kellerbauer, H.-J. Kluge, D. Lunney, R. Savreux, et al.
}

\section{- To cite this version:}

K. Blaum, D. Beck, M. Breitenfeldt, S. George, F. Herfurth, et al.. Penning trap mass spectrometry for nuclear structure studies. Hyperfine Interactions, 2006, 171, pp.83-91. 10.1007/s10751-006-9501-4 . in2p3-00147804

\section{HAL Id: in2p3-00147804 https://hal.in2p3.fr/in2p3-00147804}

Submitted on 22 May 2007

HAL is a multi-disciplinary open access archive for the deposit and dissemination of scientific research documents, whether they are published or not. The documents may come from teaching and research institutions in France or abroad, or from public or private research centers.
L'archive ouverte pluridisciplinaire HAL, est destinée au dépôt et à la diffusion de documents scientifiques de niveau recherche, publiés ou non, émanant des établissements d'enseignement et de recherche français ou étrangers, des laboratoires publics ou privés. 
Klaus Blaum • Alexander Herlert .

H.-Jürgen Kluge

\title{
Penning trap mass spectrometry for nuclear structure studies
}

Received: date / Accepted: date

\begin{abstract}
High-precision mass measurements as performed at the Penning trap mass spectrometer ISOLTRAP at ISOLDE/CERN are an important contribution to the investigation of nuclear structure. Accurate nuclear masses with less than $0.1 \mathrm{ppm}$ relative mass uncertainty not only allow stringent tests of mass models and mass formulas that are used to predict mass values of nuclides far from the valley of stability, but also to look for nuclear structure effects like shell or sub-shell closures, deformations, and halos. In addition to a sophisticated experimental setup for precise mass measurements, a radioactive ion beam facility is required that delivers a large variety of short-lived nuclides with sufficient yield. An overview of the results from the mass spectrometer ISOLTRAP is given and its limits and possibilities are described.
\end{abstract}

Keywords ISOLTRAP · mass spectrometry · nuclear structure · Penning trap $\cdot$ short-lived nuclides

PACS 7.75.+h Mass spectrometers - 21.10.Dr Binding energies and masses · 32.10.Bi Atomic masses, mass spectra, abundances, and isotopes

\section{Introduction}

Nuclear masses play an important role in a large number of physical systems [1]. Especially for nuclear structure studies and tests of mass models and

K. Blaum

Johannes Gutenberg-Universität, 55099 Mainz, Germany and GSI Darmstadt, 64291 Darmstadt, Germany

E-mail: blaumk@uni-mainz.de

A. Herlert

CERN, Physics Department, 1211 Geneva 23, Switzerland

H.-J. Kluge

GSI Darmstadt, 64291 Darmstadt, Germany and Ruprecht-Karls-Universität, 69120 Heidelberg, Germany 
formulas, nuclear binding energies of radionuclides far from the valley of stability are required [2].

Mass measurements along isotopic and isotonic chains have allowed to study the fine structure of the mass surface and clarified discontinuities in order to extract nuclear structure information such as shell and subshell closures and the onset of deformation from binding energies (see e.g. [3-7]). Along this line it was important to demonstrate that nuclear ground and isomeric states can be resolved [5, 8-12] in order to, e.g., confirm the coexistence of nuclear shapes at nearly degenerate energies $(\approx 100 \mathrm{keV})$, which had been previously deduced from laser and nuclear spectroscopy [13-15].

Mass models are available which either make estimates on unknown masses with a global approach, or try to predict locally the mass values [2]. Of course, the predictive power of these models and formulas has to be tested in regions where masses are accurately known. In most cases a mass uncertainty of 10$100 \mathrm{keV}$ is sufficient. Various experiments located at radioactive ion beam facilities aim at the investigation and precise determination of the masses of short-lived nuclides. The applied techniques range from traditional mass spectrometers to storage rings and Penning trap mass spectrometers.

However, the mass uncertainty of a large number of nuclides is above $10 \mathrm{keV}$ and the relative mass uncertainty rapidly rises the farther out the nuclides lie from the valley of stability (see Fig. 1). This is strongly correlated to the half-life and the production rates of the respective nuclides, which makes their investigation an experimental challenge.

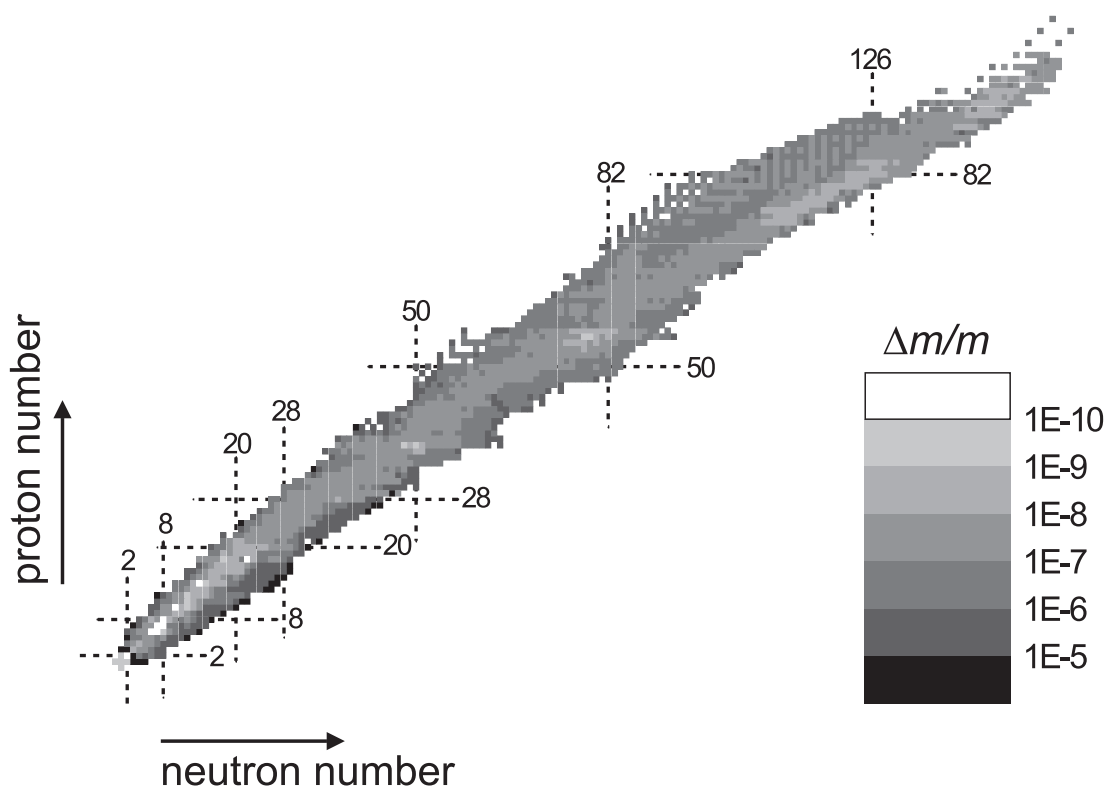

Fig. 1 Relative mass uncertainty $\Delta m / m$ of nuclides, where estimated mass excess values are omitted. Data taken from [16]. 
In this work, an outline of the ISOLTRAP Penning trap mass spectrometer and its contribution to the field of high-precision mass measurements for nuclear structure studies are presented. The possibilities, challenges, and limitations of ISOLTRAP will be discussed as well.

\section{Experimental setup and procedure}

The ISOLTRAP mass spectrometer is the prototype experiment for mass measurements of short-lived nuclides with a Penning trap. Various additional experiments are meanwhile in operation or under construction. For a comprehensive review see [1]. A schematic overview of the experimental setup is shown in Fig. 2. The radioactive ions are delivered from one of the two target stations at ISOLDE. The short-lived nuclides are produced by bombarding a fixed target with a $1.4 \mathrm{GeV}$ proton pulse. Due to spallation, fragmentation, and fission a large number of radionuclides is formed. The target container is heated and the radioactive particles diffuse into an ion source. Three types
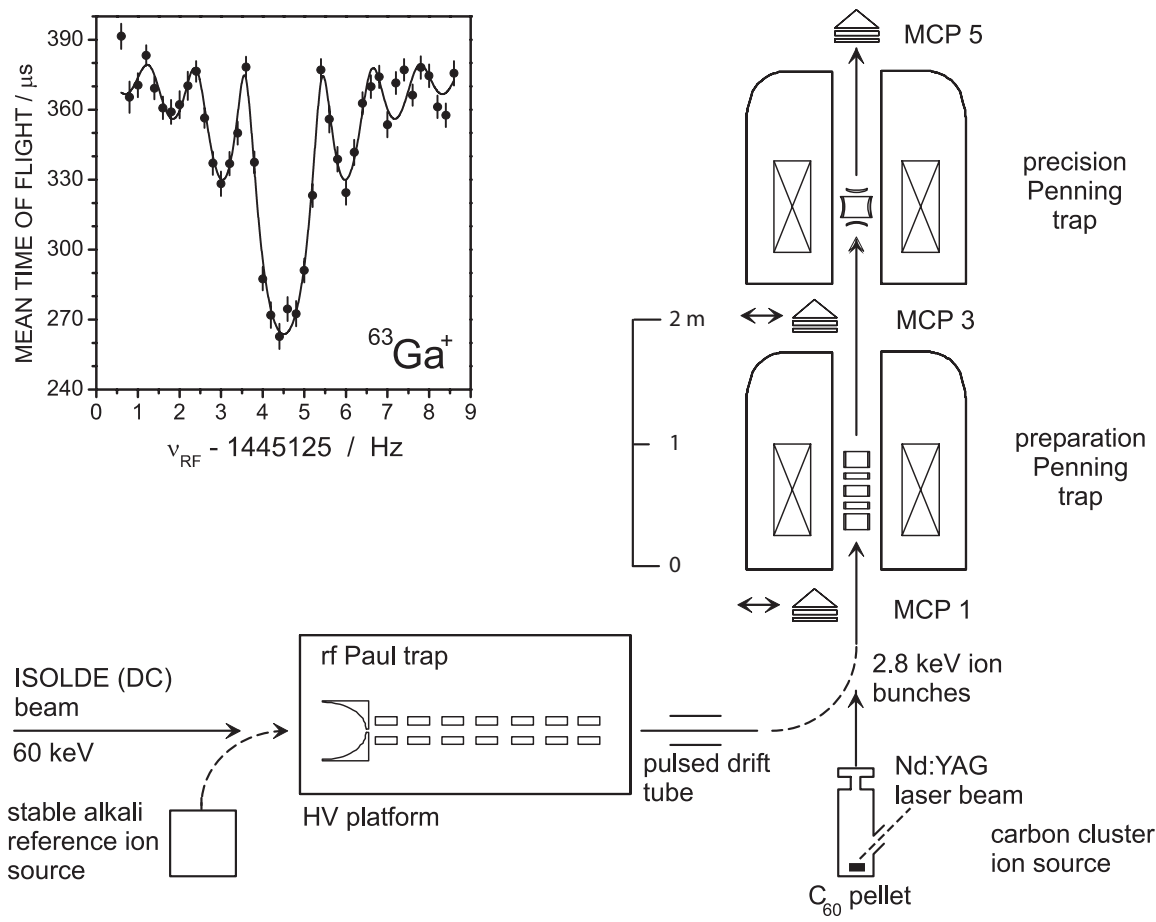

Fig. 2 General layout of the ISOLTRAP experiment. The main components are a radiofrequency quadrupole ion beam cooler and buncher, a preparation Penning trap for isobaric cleaning of the radioactive ion ensemble, and a $5.9 \mathrm{~T}$ Penning trap mass spectrometer. Micro-channel-plate (MCP) detectors are used to monitor the ion transfer as well as to record the time-of-flight resonance (MCP5) for the determination of the cyclotron frequency. The inset shows a cyclotron resonance curve for the short-lived radionuclide ${ }^{63} \mathrm{Ga}^{+}\left(T_{1 / 2}=31.4 \mathrm{~s}\right)$ with $900 \mathrm{~ms}$ duration of the quadrupolar rf excitation in the precision Penning trap. 
of ion sources are available, a surface ion source, a resonant laser ion source, and a (hot or cold) plasma ion source. After passing separator magnets the usually singly charged ions are sent as a $60-\mathrm{keV}$ continuous beam to the ISOLTRAP setup.

By use of a linear radiofrequency quadrupole structure [17], filled with helium buffer gas, and mounted inside a $60-\mathrm{keV}$ high voltage cage, the continuous beam is stopped, accumulated, and bunched as well as cooled for the further injection into the first Penning trap. This cylindrically shaped trap also employs a helium buffer gas and a sophisticated cooling technique is applied to mass-selectively center only the radionuclide ions of interest by use of a quadrupolar rf excitation [18]. Thus unwanted isobaric contaminant ions can be removed with a resolving power of $R=10^{4}-10^{5}$ depending on the respective mass and excitation duration [19].

The cooled, isobaric pure ion bunch is finally transferred to the hyperbolically shaped precision Penning trap, where the cyclotron frequency

$$
\nu_{c}=\frac{1}{2 \pi} \frac{q B}{m}
$$

of the stored ions with mass $m$ and charge $q$ moving in a magnetic field $B$ is probed after some preparatory steps [20] with a destructive time-offlight cyclotron resonance detection technique $[21,22]$. In order to deduce the mass of the investigated radionuclide ions, the magnetic field strength $B$ needs to be determined at the same level of precision. This is done via a frequency measurement of a well-known stable nuclide ion either delivered from the ISOLTRAP alkali ion source or the ISOLDE target. By calculating the frequency ratio, the mass ratio is obtained. Note that the mass of missing electrons has to be taken into account in the analysis.

\section{Recent results at ISOLTRAP}

\subsection{Nuclear structure studies}

The contribution of mass measurements to nuclear structure studies is based on the possibility to determine the nuclear binding energy

$$
B(N, Z)=\left(N m_{n}+Z m_{p}-m(N, Z)\right) c^{2},
$$

which represents the sum of all the nucleonic interactions that give rise to correlations in many-body systems. Since the binding energy depends on the detailed composition of protons and neutrons, the mass of each of the more than 3000 nuclides as observed to date [16] is highly specific and a key property of a nuclear system. By taking a closer look at this ensemble of mass data covering the whole nuclear chart, one can examine the hills and valleys that form this mass surface and make hypotheses about the effects of certain nuclear configurations. To unveil these effects, mass measurements with an accuracy of $\delta m / m<10^{-6}$ are required.

Mass differences lead to separation energies, i.e., the energy needed to separate some nucleons from the nucleus, providing information on the shell 
structure and phase transitions. The most striking way in which the shell structure manifests itself in mass systematics is through double differences of masses, i.e., the two-neutron separation energy

$$
S_{2 n}(N, Z)=B(N, Z)-B(N-2, Z)
$$

in the case of the neutron shells, and the two-proton separation energy

$$
S_{2 p}(N, Z)=B(N, Z)-B(N, Z-2)
$$

in the case of proton shells. Here, specific classes of interactions can be isolated [2]. The single-nucleon separation energy is a less clear-cut indication because of the pairing effect.

In general, $S_{2 n}$ decreases as neutrons are added and a deviation from this behavior points to manifestations of microscopic nuclear structure effects, as it has been, e.g., observed with ISOLTRAP in the $\mathrm{Hg}$ and $\mathrm{Pt}$ region [5]. Similar investigations have been performed recently in the neutron-rich Ni$\mathrm{Cu}-\mathrm{Ga}$ mass region $[23,24]$

\subsection{Resolution of isomeric states}

An important issue in direct mass measurements with respect to nuclear structure studies is to resolve isomeric and ground states, since nearly one third of the nuclides in the nuclear chart have long-lived isomeric states with, in many cases, unknown excitation energies. In the context of nuclear physics, isomers are excited states of atomic nuclides at excitation energies from $100 \mathrm{keV}$ up to a few $\mathrm{MeV}$. Their half-lives range from nanoseconds to beyond the age of the Universe, e.g. $10^{15}$ years in the case of ${ }^{180 m} \mathrm{Ta}$, the only naturally occurring isomer on Earth [25].

Direct high-accuracy mass measurements of isomeric states with ion traps have already been performed several times. The very first isolation of an isomeric state in a Penning trap was demonstrated by G. Bollen et al. in 1992 [8]. In that work, the ${ }^{84 m} \mathrm{Rb}$ isomer could be eliminated due to its shorter half-life as compared to the ground state by simply storing the ensemble of nuclide ions for a sufficiently long period in the trap. In the case of ${ }^{78} \mathrm{Rb}$, for which this approach was impossible, the large resolving power of the Penning trap enabled a direct resolution of the two states ${ }^{78} \mathrm{Rb}$ and ${ }^{78 m} \mathrm{Rb}$, separated by an energy $E=111.2 \mathrm{keV}$. In a series of measurements, several isomeric excitation energies were determined for some odd-mass mercury isotopes ${ }^{185 m} \mathrm{Hg},{ }^{187 m} \mathrm{Hg},{ }^{191 m} \mathrm{Hg},{ }^{193 m} \mathrm{Hg}$, and ${ }^{197 m} \mathrm{Hg}$ [5]. In these cases an extremely high resolving power of up to $3.7 \cdot 10^{6}$ was applied, corresponding to excitation times of up to $8 \mathrm{~s}$. Recently, the energy difference of ${ }^{187} \mathrm{~Pb}$ and ${ }^{187 m} \mathrm{~Pb}$ was determined with Penning trap mass spectrometry to be $E=$ $33(13) \mathrm{keV}$ [12]. This is the lowest isomeric excitation energy ever determined by weighing nuclei. The most intricate example is the one of ${ }^{70} \mathrm{Cu}$, for which a simultaneous combination of laser ionization, decay spectroscopy, and mass spectrometry succeeded in assigning the three low-lying states, the ground state and the two excited isomers ${ }^{70 m} \mathrm{Cu}$ and ${ }^{70 n} \mathrm{Cu}$, with excitation energies $E_{m}=100.7(2.6) \mathrm{keV}$ and $E_{n}=242.0(2.7) \mathrm{keV}$, respectively, to the correct spin values [11]. 
3.3 Test of nuclear mass models and mass formulas

The nucleus is a self-organized, many-body quantum system that interacts through the strong, weak and electromagnetic forces. Due to the lack of an exact description of the strong interaction and the complexity of the many-body nucleonic system, the binding energy can not be described by ab-initio theories. Instead, one has to rely on mass predictions by models (with the aim of a quantitative prediction of the total binding energy of a nucleus) and formulas (with the aim of a numerical calculation of masses on a physical basis) [2]. For decisive tests of the predictive power of the different models, large-scale mass measurements of exotic short-lived nuclei have been performed in recent years, especially at the experimental storage ring ESR at GSI-Darmstadt $[9,26,27]$ but also at ISOLTRAP $[3-5,23,28]$. Surely, highaccuracy mass data will contribute to further improvement and refinement of mass models and formulas and to more detailed understanding of the nuclear forces.

Aside from global mass formulas, there is also a number of local mass formulas, which address the problem to obtain the required mass of a so far unmeasured nucleus that lies fairly close to a considerable number of nuclei with known masses. Stringent tests of one of the most powerful local mass formula, the isobaric multiplet mass equation (IMME) [29], have been performed at ISOLTRAP in recent years. In light nuclei, isobaric analog states (IAS) have nearly identical wave functions. The charge dependent energy difference of these states can be calculated using first-order perturbation theory assuming only two-body Coulomb forces. This leads to the simple equation, noted first by Wigner [30] and Weinberg and Treiman [31], that gives the mass $m$ of a member of an isospin multiplet as a function of its isospin projection $T_{Z}=(N-Z) / 2$ :

$$
m\left(T_{Z}\right)=c_{0}+c_{1} T_{Z}+c_{2} T_{Z}^{2}
$$

This quadratic relation of the IMME is of fundamental importance in isospin symmetry in nuclear physics [32]. Looking at the isospin quartets [29] it was found that the IMME worked very well for 21 out of 22 cases. Due to its success and lack of newer experimental data, the IMME is widely used to predict masses as well as level energies, for example for the mapping of the proton drip line over a wide mass range, which is important for determining the $r p$-process path.

In 2001 the ISOLTRAP mass value for ${ }^{33} \mathrm{Ar}$ resulted in a breakdown of the quadratic IMME for the $A=33, T=3 / 2$ quartet [33]. These surprising results triggered new reaction spectroscopy experiments. The outcome was that one level energy in ${ }^{33} \mathrm{Cl}$ was inaccurate [34], which shows the importance of refined measurements. Of course, a direct mass measurement can only determine the mass of ground state multiplet members. But these nuclei are often also the most exotic members of an isospin multiplet with a rather large mass uncertainty [32].

To test the IMME with an accuracy never obtained before, ISOLTRAP performed mass measurements on the very short-lived nuclides ${ }^{32} \mathrm{Ar}\left(T_{1 / 2}=\right.$ $98 \mathrm{~ms})$ and ${ }^{33} \mathrm{Ar}\left(T_{1 / 2}=173 \mathrm{~ms}\right)$ and used the mass excess values of the other 
states of the $T=2$ quintet for $A=32$ and the $T=3 / 2$ quartet for $A=33$ [35]. For the test of the IMME, an additional cubic term $c_{3} T_{Z}^{3}$ is assumed in Eq. (5) that should fit with $c_{3}=0$ if the quadratic form of the IMME is correct. The experiment yielded a coefficient $c_{3}=-0.11(30)$ in the case of $T=2, A=32$ and $c_{3}=-0.13(45)$ in the case of $T=3 / 2, A=33$. Thus, in both cases the $c_{3}$ coefficient is consistent with zero within the uncertainty. These two multiplets now represent the most stringent test of the IMME. Even more decisive tests should come soon.

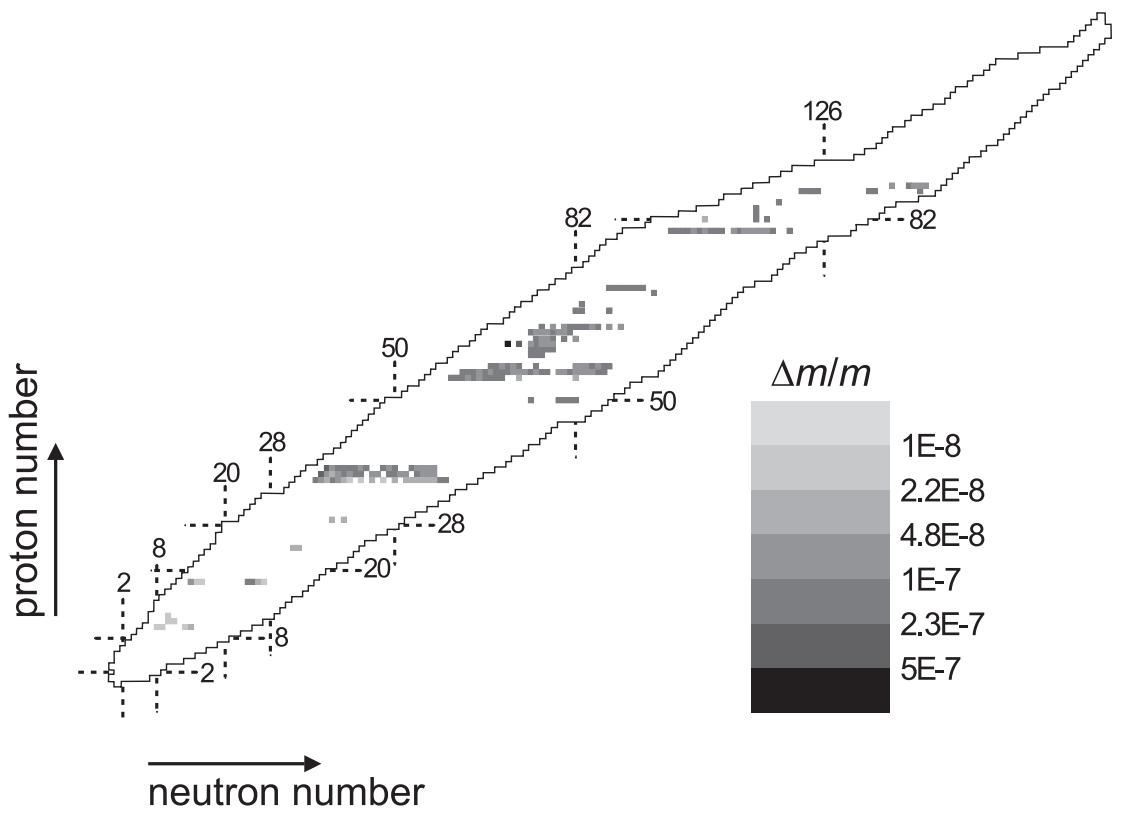

Fig. 3 Relative mass uncertainty of published mass values obtained at ISOLTRAP.

\section{Present status and technical development at ISOLTRAP}

In the past, ISOLTRAP has measured the mass of about 300 nuclides. The relative mass uncertainty resulting from so far published frequency ratio values measured at ISOLTRAP are shown in Fig. 3. While in the beginning the systematic deviations of the spectrometer were not known and therefore a limitation to a relative mass uncertainty in the order of $1 \times 10^{-7}$ was given, the systematic investigation of the setup with the mass measurement of carbon clusters was a major improvement [36]. Carbon clusters consisting of a multiple of ${ }^{12} \mathrm{C}$ atoms are the ideal reference masses, because the atomic mass unit is defined with respect to the mass of ${ }^{12} \mathrm{C}$ [37]. A systematic study showed a limitation in the accuracy of ISOLTRAP of $\delta m / m=8 \times 10^{-9}$ [38], which is added as a systematic error to the statistical error from the frequency determination. Nowadays results regularly reach down to relative 
mass uncertainties in the order of $1 \times 10^{-8}$, some of them are also shown in Fig. 3.

Recent technical improvements at the ISOLTRAP mass spectrometer include a new ion detector and a magnetic field stabilization of the superconducting magnet of the precision Penning trap. The existing micro channel plate (MCP) detector was replaced by a channeltron detector with a conversion dynode, where secondary electrons are monitored with an efficiency close to $100 \%$. With this setup an increase of the total efficiency by a factor of about 3 was obtained and nuclides with a lower production yield can be addressed [39].

The systematic uncertainty of the mass measurements can be improved with a stabilization of the magnetic field. As has been observed recently, the cyclotron frequency of the stored ions depends on the temperature in the warm bore of the superconducting magnet [40]. A new stabilization system has been installed which aims at a stabilization of this temperature with variations of less than $\pm 10 \mathrm{mK}$.

Finally, a new technique to obtain radionuclides that are not delivered from ISOLDE has been tested: the production of radionuclides by in-trap decay [41]. To this end, short-lived nuclides are first stored in the buffer-gas filled preparation Penning trap and after a respective storage period, enough daughter nuclides from the decay will be collected in the trap. After the usual mass-selective cooling and centering procedure, they are transferred to the precision trap for mass determination. This technique has been successfully applied to produce ${ }^{61,62,63} \mathrm{Fe}$ isotopes, which are not available as an ISOLDE beam, and to measure their mass. The results are currently under investigation.

\section{Conclusion and outlook}

The ISOLTRAP mass spectrometer at ISOLDE is a source for accurate mass values of short-lived radionuclides, where the relative mass uncertainty is currently limited to $\delta m / m=8 \times 10^{-9}$ [38]. With the possibility to reach nuclides with half-lives well below $100 \mathrm{~ms}$ [42] and production yields down to 100 ions per second [35], a large number of radionuclides have been and can be investigated at ISOLDE.

Besides target development at ISOLDE, which aims at higher yields, more accessible elements, and less contamination, the experimental setup of ISOLTRAP is constantly subject of technical development. A new ion detector for a better overall efficiency, a stabilization of the magnetic field, and new ion sources that deliver a variety of so far not applicable reference masses are recent improvements of the setup. Further development is directed to a higher precision in the frequency determination by use of new rf excitations schemes [43] or highly charged ions [44].

Acknowledgements This work was supported by the German Ministry for Education and Research (BMBF) under contract 06GF151, by the European Commission under contracts HPMT-CT-2000-00197 (Marie Curie Fellowship), HPRICT-2001-50034 (NIPNET), and RII3-CT-2004-506065 (TRAPSPEC), and by the 
Helmholtz association of national research centres (HGF) under contract VH-NG037. We also thank the ISOLDE Collaboration as well as the ISOLDE technical group for their assistance.

\section{References}

1. K. Blaum, Phys. Rep. 425 (2006) 1.

2. D. Lunney, J.M. Pearson, and C. Thibault, Rev. Mod. Phys. 75 (2003) 1021.

3. F. Ames et al., Nucl. Phys. A 651 (1999) 3.

4. D. Beck et al., Eur. Phys. J. A 8 (2000) 307.

5. S. Schwarz et al., Nucl. Phys. A 693 (2001) 533.

6. S. Rinta-Antila et al., Phys. Rev. C 70 (2004) 011301(R).

7. Yu.A. Litvinov et al., Nucl. Phys. A 756 (2005) 3

8. G. Bollen et al., Phys. Rev. C 46 (1992) R2140.

9. Yu.A. Litvinov et al., Nucl. Phys. A $\mathbf{7 3 4}$ (2004) 473.

10. K. Blaum et al., Europhys. Lett. 67 (2004) 586.

11. J. Van Roosbroeck et al., Phys. Rev. Lett. 92 (2004) 112501.

12. C. Weber et al., Phys. Lett. A 347 (2005) 81.

13. E.W. Otten, Treatise on heavy-ion science, 8, ed. D. Allan Bromley, Plenum Press, New York, 515 (1989).

14. A.N. Andreyev et al., Nature 405 (2000) 430.

15. H.-J. Kluge, W. Nörtershäuser, Spectrochim. Acta B 58 (2003) 1031.

16. G. Audi, A.H. Wapstra, and C. Thibault, Nucl. Phys. A $\mathbf{7 2 9}$ (2003) 337.

17. F. Herfurth et al., Nucl. Instrum. Meth. A 469 (2001) 254.

18. G. Savard et al., Phys. Lett. A 158 (1991) 247.

19. H. Raimbault-Hartmann et al., Nucl. Instrum. Meth. B 126 (1997) 378.

20. K. Blaum et al., J. Phys. B: At. Mol. Opt. Phys. 36 (2003) 921.

21. G. Gräff, H. Kalinowsky, and J. Traut, Z. Phys. A 297 (1980) 35.

22. G. Bollen et al., Nucl. Instrum. Meth. A 368 (1996) 675.

23. C. Guénaut et al., J. Phys. G: Nucl. Part. Phys. 31 (2005) S1765.

24. C. Guénaut et al., Eur. Phys. J. A 25 (2005) 33.

25. G. Audi et al., Nucl. Phys. A 729 (2003) 3.

26. Yu.A. Litvinov et al., Hyperfine Interactions 132 (2001) 283.

27. Yu. N. Novikov et al., Nucl. Phys. A 697 (2002) 92.

28. P. Delahaye et al., Phys. Rev. C 74 (2006) 034331.

29. W. Benenson, E. Kashy, Rev. Mod. Phys. 51 (1979) 527.

30. E.P. Wigner, in: W.O. Millikan (Ed.), Proceedings of the Robert A. Welch Foundation Conference on Chemical Research, Houston, vol. 1, Robert A. Welch Foundation, Houston, 1957.

31. S. Weinberg, S.B. Treiman, Phys. Rev. 116 (1959) 465.

32. J. Britz, A. Pape, M. Antony, At. Data Nucl. Data Tab. 69 (1998) 125.

33. F. Herfurth et al., Phys. Rev. Lett. 87 (2001) 142501.

34. M.C. Pyle et al., Phys. Rev. Lett. 88 (2002) 122501.

35. K. Blaum et al., Phys. Rev. Lett. 91 (2003) 260801.

36. K. Blaum et al., Eur. Phys. J. A 15245 (2002).

37. K. Blaum et al., Anal. Bioanal. Chem. 3771133 (2003).

38. A. Kellerbauer et al., Eur. Phys. J. D 22 (2003) 53.

39. C. Yazidjian et al., accepted in Hyperfine Interactions (2006).

40. K. Blaum et al., J. Phys. G: Nucl. Part. Phys. 31 (2005) S1775.

41. A. Herlert et al., New J. Phys. 7 (2005) 44.

42. A. Kellerbauer et al., Phys. Rev. Lett. 93 (2004) 072502.

43. S. George et al., to be submitted to Int. J. Mass Spectrom. (2006).

44. A. Herlert et al., Int. J. Mass Spectrom. 251 (2006) 131. 